\title{
How can perceptual experiences explain uncertainty?
}

\section{Susanna Siegel}

Department of Philosophy, Harvard University, Cambridge, Massachusetts

\section{Correspondence}

Susanna Siegel, Department of Philosophy, Emerson Hall, Harvard University, Cambridge, MA 02138.

Email: ssiegel@fas.harvard.edu
Can perceptual experiences be states of uncertainty? We might expect them to be, if the perceptual processes from which they are generated, and the behaviors they help produce, take account of probabilistic information. Yet, it has long been presumed that perceptual experiences purport to tell us about our environment, without hedging or qualifying. Against this longstanding view, I argue that perceptual experiences may well occasionally be states of uncertainty, but that they are never probabilistically structured. I criticize a powerful line of reasoning that we should expect perceptual experience to be probabilistic, given their interfaces with unconscious probabilistic information, with behavior responsive to it, and with credences.

\section{KEYWORDS}

Bayesianism, consciousness, credences, perception, prediction, probability

\section{1 | INTRODUCTION}

Our languages are full of ways to express uncertainty, such as "I suspect that ...," "I am more sure of X than I am of Y," "I cannot rule out X but I cannot rule it in either." You might feel doubtful that the cries you heard in the desert were a baby (since coyotes can sound like that), yet not entirely comfortable ruling out this eerie possibility.

Some hold that degrees of confidence are probabilistically measurable. Let us call these degrees of confidence credences. If you have a middling credence in $p$, you are far from fully confident in accepting or rejecting it. To express your opinion on $p$, you would have to hedge, by using one of the many locutions we have for qualifying an outright "Yes" or "No."

Credences are states of uncertainty. How similar can they be to perceptual experience? If I am not completely sure that the cries in the desert come from a coyote, can this be because my 
perceptual experience already embodies a form of uncertainty intrinsically, or must my uncertainty be merely a contingent response to my perceptual experience?

Uncertainty intrinsic to perceptual experience would supervene on the phenomenal character of the experience. Anyone having a perceptual experience with that phenomenal character would undergo that same form of uncertainty. As I am using "perceptual experience," perceptual experience always feels like perception, which makes it a phenomenal category. ${ }^{1}$ Phenomenal character is intrinsic to experience. I will sometimes call it "experience" for short. If perceptual experience can ever be a state of uncertainty, then there is a form of uncertainty that is intrinsic to perceptual experience.

To illustrate uncertainty intrinsic to perceptual experience, consider the following argument for it:

\section{Face-value argument}

(P1) You can form credences by taking an experience at face value.

(P2) If you can form credences by taking an experience at face value, then the experience must be intrinsically a state of uncertainty.

(Conclusion): Experiences can be intrinsically states of uncertainty.

This argument develops the idea that if the phenomenal character of your experience alone could guide you into a state of postperceptual uncertainty, then your experience must already be its own form of uncertainty.

According to the conclusion of the face-value argument, the phenomenal character of experience is non-contingently related to uncertainty. A stronger, explicitly probabilistic version of this claim would be that the phenomenal character of experience can be non-contingently related to a specifically probabilistic form of uncertainty-one we might call perceptual protoconfidence, since it would be a perceptual precursor to confidence.

Both the conclusion and the stronger claim allow that the phenomenal character of perceptual experience non-contingently attaches a measure of uncertainty to information about the way things are in the environment. ${ }^{2}$ We can say that when an experience does this, it is a phenomenal hedge-marker. If experience is a probabilistic phenomenal hedge marker, then you could read off probabilistic information from the phenomenal character, as much and in the same way as you could read off any other information that can be noncontingently related to phenomenal character, such as whether there is anything red to your left.

In this paper, I argue that taken on its own, the conclusion of the face-value argument may well be true, but that both premise (P1) and the probabilistic version of its conclusion are false. Some experiences are states of uncertainty, but none of them are probabilistic: Phenomenal character does not inherit probabilistic information from the perceptual processing that produces it; it does not convey probabilistic information to subsequent states; and it is not

\footnotetext{
1“Perceptual experience" can alternatively be heard as denoting whatever experience accompanies perception, as opposed to the phenomenological category of experiences that feel like perception, whether they are perceptions of external stimuli or not. My use is the phenomenological reading. The two uses differ because as Perky (1910) showed, experiences that accompany perception may feel like imagination.

${ }^{2} \mathrm{~A}$ hedge on whether a worldly condition C obtains can take the form of probabilistic "worldly" contents that assign a probability to condition $\mathrm{C}$ obtaining, or to probabilistic meta-representational contents that assign a probability to the correctness of a content that says $\mathrm{C}$ obtains. I will count both kinds of contents as a hedge on $\mathrm{C}$, even though they will be psychologically different.
} 
probabilistically structured. If experiences can be states of uncertainty, such states are few and far between, and contrary to (P1) it is not possible to form probabilistically measurable levels of confidence directly on the basis of experience.

With a small adjustment, the face-value argument could be modified to yield the stronger probabilistic conclusion that I am arguing against:

\title{
Probabilistic face-value argument
}

(P1) You can form credences by taking an experience at face value.

(P2*) If you can form credences by taking an experience at face value, then the experience must be intrinsically probabilistic.

(Conclusion) Experiences can be intrinsically probabilistic.

Morrison (2015) offers an argument much like this revised one. ${ }^{3}$ In both face-value arguments, a host of challenges surrounds the premises, and recent discussions have made many of these challenges evident. ${ }^{4}$

Here, I assess the idea that experiences are intrinsically probabilistic by engaging with a different route to that conclusion that avoids the complexities of the face-value arguments and instead draws heavily on perception science. Its conceit is that in cases where behavior is sensitive to probabilistic information that is used in perceptual processing, we should expect that probabilistic information to be conveyed by perceptual experience as well.

For instance, Morrison (2019) describes the informational continuity he thinks we should expect to find reaching from subpersonal processing, through experience, to behavior:

\begin{abstract}
Suppose we can show that degrees of confidence that are assigned by early perceptual processing are also available for behavior. This is a reason to think that the relevant degrees of confidence are assigned by the perceptual experience itself. If that degree of confidence didn't arise until after the perceptual experience, we wouldn't expect to find it in the brain earlier than perceptual experience. And if that degree of confidence were discarded, we wouldn't expect to find the same degree of confidence later. Once information is lost, it cannot be regained. (Morrison, 2019)
\end{abstract}

Probabilistic experiences, on this picture, are part of a continuous flow of probabilistic information from subpersonal perceptual processing to producing probabilistically guided behavior. Such experiences safeguard against losing perceptual information along the way to behavior that they guide. And the main alternative to probabilistic experiences, Morrison goes on to suggest, is that experiences would be epiphenomenal with respect to probabilistic behavior-a consequence he rejects as implausible.

After some preliminary discussion in Section 2, in Sections 3 and 4, I present and attempt to undermine the argument for probabilistic experiences from considerations about the relationship between probabilistic processing and perceptual experience. I then turn to assessing directly whether there are probabilistic experiences. My strategy is to

\footnotetext{
${ }^{3}$ Morrison (2019) formulates his argument in terms of trust when he writes that probabilistic experiences "best explains what happens when we completely trust our experience."

${ }^{4}$ Beck (2019), Cheng (2018), Pohl (2019), Nanay (2020), and Raleigh and Vindrola (n.d.). Unlike these writers, I will not discuss $\mathrm{P} 2$ at all.
} 
begin by considering the more fundamental claim that that experiences can be intrinsically states of uncertainty. This claim, as we saw, is the conclusion of the unmodified face-value argument, and I make the case right at the start that it is interesting and important in its own right, as it challenges long-standing and deeply entrenched assumptions about the nature of perceptual phenomenology. I argue that experiences may well be intrinsically states of uncertainty (Section 5), but that they are not probabilistically structured (Section 6).

\section{2 | WHY DOES IT MATTER IF EXPERIENCES CAN BE STATES OF UNCERTAINTY?}

What would it be for a perceptual experience to be a state of uncertainty, and why does it matter whether any of them are?

In a state of uncertainty, one hedges on whether something is or is not the case. A hedgemarker is something in the mind that marks off a content as a way things in the environment might but might not actually be. ${ }^{5}$ A probabilistic structured, middling level of confidence is a paradigmatic hedge-marker, but not the only kind.

A phenomenal hedge-marker is a phenomenal feature of perceptual experience that marks off a content as a way that things in your environment might but might not actually be. In phenomenal hedge-markers, phenomenal character attributes uncertainty to information about the external environment.

How can a phenomenal hedge-marker attach uncertainty to external-world information by virtue of its phenomenal character? We can distinguish between two kinds of phenomenal hedge-markers: intrinsic and role-based. Intrinsic phenomenal hedge-markers mark the hedge with intrinsic features of experiences, whereas role-based phenomenal hedge-markers mark the hedge by one or more roles played by phenomenal character, where the roles are only contingently associated with the experience. For instance, if high pitched sounds scare you, then a role played by your auditory experience is to cause fear. However, this role is only contingently related to the auditory experience, since someone might have the same experience without feeling scared, and you might cease to feel scared by such sounds after further conditioning, for instance if you listen to a lot of operas. Similarly, if an experience is a role-based phenomenal hedge-marker, the experience causes you to hedge on a content, but its doing that is a contingent feature of the experience.

If there are role-based phenomenal hedge-markers, then experiences are not necessarily states of uncertainty. If there are intrinsic phenomenal hedge-markers, then by definition, some experiences are states of uncertainty-those with phenomenal features that constitute hedgemarkers.

We can understand what the intrinsic variety of hedge-marker would be by considering what it would be for intrinsic features of experiences to constitute a hedge.

\footnotetext{
${ }^{5}$ The type of contents relevant to this discussion are accuracy conditions. As Morrison (2015) points out, if experiences were structured in the same way as credence distributions over a hypothesis set, with perceptual seeming in place of credence relations to each hypothesis, then the experience as a whole would not have accuracy conditions in any straightforward sense. However, we can still think of contents as the hypotheses, and that is the sense of "content" at issue here.
} 
The intrinsic features of experience include its phenomenal character and phenomenal contents of experience (i.e., contents that supervene on phenomenal character). ${ }^{6}$ For instance, suppose the phenomenal contents of experience included probabilistic contents, such as "there's probably a cat in front of me." Encoding this probabilistic information would be but one way for experiences to be states of uncertainty. If an experience encoded probabilistic information in its phenomenal character, then any two phenomenally identical experiences would encode the same probabilistic information.

A different example of an intrinsic feature of experience is the invariant phenomenal features of experience. Perceptual experience, as noted earlier, always feels like perception, whether it is a case of perceiving or not. It is a phenomenal category with invariant features that are shared by all perceptual experiences, whether those experiences are cases of perceiving or something else, such as imagining. Hypotheses about the invariant features of perceptual experience are universal claims about all perceptual experiences. ${ }^{7}$

Perception itself includes more than perceptual experience. Perceiving can feel like imagining, as Perky showed long ago. And perceptual experiences are not always cases of perception, as is shown by immersive dreaming and hallucinating. ${ }^{8}$

Finally, the structure of a perceptual experience could be a hedge-marker, if by virtue of that structure, the experience presents us with multiple incompatible possibilities at once, and marks the possibilities as a way the world might actually or might not actually be. Section 5.1 makes the case that such "multiplex" experiences are the best candidates for intrinsic phenomenal hedge-markers, and Section 6 argues that these experiences are not probabilistic: There is no intrinsic assignment of probabilistic weights to each option.

\subsection{The standard view of perceptual phenomenology}

A first reason why it matters whether perceptual experiences can be states of uncertainty is that intrinsic phenomenal hedge-markers are not supposed to exist, according to a longstanding view of the phenomenology of perception.

According to the longstanding view, which I will call the standard view, an invariant feature of perceptual experience is that it purports to present us with a unique way that things actually are at the time of the experience. This position is often defended as part of a scheme for differentiating the invariant phenomenal features of perception, imagination, and episodic memory. ${ }^{9}$

\footnotetext{
${ }^{6}$ I will leave it open whether all phenomenal features of experience are also representational. Vance (2020), like Block (1996), suggests that some aspects of phenomenal character are manners of representation of contents. Vance proposes that precision information in perceptual processing explains some manners of representation, such as blur, but leaves open whether blur is intrinsically a hedge-marker.

${ }^{7}$ There are also arguably such things as phenomenal imagining and phenomenal remembering, and for each of these phenomenal states, we could ask what its phenomenal invariant features are. However, here I am concerned only with perceptual experience and whether it can be a phenomenal hedge-marker. Madary (2012) discusses a related question. ${ }^{8}$ Perky (1910). Imagining, in turn, can feel like remembering, as we know from studies of false memories (Mazzoni \& Memon, 2003; Schacter \& Dodson, 2001), just as remembering can feel like imagining, as Martin and Deutscher observed when they developed their causal theory of memory (Martin \& Deutscher, 1966). On the phenomenology of dreams see Hobson (2005). ${ }^{9}$ I call the view "standard" because thinkers from different corners of philosophy and neuroscience converge on it from different places and times, including Husserl (2005) on memory versus "phantasy," Lau (2019) on perception as reality monitoring, and Martin (2002) on perception versus imagination. Husserl's terminology does not map on to mine, since he draws finer distinctions that recognize intermediate states between what someone might call perception and memory (such as retention) and between perception and imagination (protention). Husserl's term for the experiences that have the phenomenology of perception described here is "adumbration." Note that Figure 1 leaves open whether perceptual experiences are or involve representational states.
} 
According to the standard view, if you phenomenally imagine an apple in front of you, you can experience being in the presence of an apple as a merely possible way things could be, or even a way things could be in the future or could have been. Phenomenally, this type of experience is different from episodically remembering seeing an apple. In that case, even if you picture an apple vividly in your mind, the experience is marked as something that actually occurred in the past, rather than as something imaginary that is not actually happening now.

On the standard view, phenomenal perception contrasts with phenomenal memory and phenomenal imagination by presenting things as actually occurring at the moment of the experience, rather than as having actually having occurred in the past (as per phenomenal memory) or as not actually occurring presently at all (as per phenomenal imagination). On this view, there is no way to "hedge" in perceptual experience, no way to mark off something we are perceptually conscious of as merely possible, the way we do in imagination and in thought.

Since the standard picture is about invariance in perceptual experience, it makes universal predictions about all perceptual experiences. So if there are intrinsic phenomenal hedgemarkers, then the standard picture is wrong. I argue in Section 5 that the standard picture may well be unable to accommodate some perceptual experiences of wobbly trajectories, and I suggest a different scheme for distinguishing between phenomenal perception, phenomenal imagination, and phenomenal memory that can accommodate these odd experiences.

\section{2 | How can we discover whether there are intrinsic phenomenal hedge-markers?}

A second reason to find out whether perceptual experiences can be states of uncertainty is that the answer to this question bears on how models from perception science may map on to visual phenomenology.

Not all information processed by our perceptual system ends up in our perceptual experience. If perceptual experiences can be states of uncertainty, then the discovery of such experiences contributes to the science of consciousness by clarifying the options for how models in perception science may map on to perceptual experience.

How can we discover whether any experiences are states of uncertainty? A method suggested by Denison, Block, and Samaha (2020) is to start from models of the features detected in early vision that posit various forms of uncertainty in perceptual processing, such as signal detection theory, drift diffusion models, probabilistic population codes, or sampling, and then try to figure out which components of those models explain any phenomenal features of perceptual experience, such as experiences of orientation or the direction of motion. If any of the components that explain phenomenology involve uncertainty, uncertainty in one way or another will be reflected in experience. Once a model is successful at explaining behavioral data, further experiments can sometimes help rule out some hypotheses about which information represented by the model gets inherited by experience. ${ }^{10}$

\footnotetext{
${ }^{10}$ On their own, none of the models discussed by Denison et al. determine which components of the model maps to phenomenology and which do not. For instance, drift diffusion models posit accumulators that gather evidence and reach a perceptual decision when the accumulated evidence crosses a threshold, but the decision threshold can be set in different places depending on other factors. Once evidence accumulates to the decision threshold amount, the perceptual system represents a feature, such as leftward direction of motion. If the phenomenal character of visually experiencing leftward motion is sensitive to whether the threshold is reached, then a stimulus will flit in and out of visibility as the decision threshold moves, even if the amount of accumulated evidence stays the same. As Denison et al. point out, so long as the decision variable can be controlled and the evidence accumulated can be measured, it is possible to test this hypothesis.
} 
This approach to the science of consciousness involves reasoning about what else would be true of experience, if one or another mechanism posited by the model for perception of an individual feature explained perceptual experience of that feature. There is no single method or set of experiments underlying this approach. Instead, what is distinctive is the inquiry into what else would be true of the experiences of a feature, given assumptions about which part of the model explains the experience.

This approach has to navigate the enduring methodological challenge that since consciousness involves a subject's perspective, it is hard to avoid relying on subjects' reports, yet it is also difficult to determine whether subjects' reports are reporting perceptual experience or merely judgments made in response to them. This obstacle is not necessarily insurmountable, but it is one place where philosophical analyses of phenomenology may help.

A different challenge is specific to the search for phenomenal uncertainty. While it may seem clear enough what phenomenal character experiences of orientation or direction of movement have, it is much less obvious what perceptual experiences of uncertainty might consist in. Understanding the nature of phenomenal uncertainty in turn clarifies hypotheses about which types of uncertainty posited by models could be mapped onto phenomenology, by clarifying the target of such mappings. ${ }^{11}$

A third reason to try and discover whether experiences can be states of uncertainty is that it bears on the roles of experiences in explaining behavior. As we will see next, a powerful argument for probabilistic experiences depends on hypotheses about the explanatory relationships between experiences, the sensory systems that produce them, and behavior that is responsive to uncertainty.

\section{3 | IF SOME BEHAVIOR IS RESPONSIVE TO PROBABILISTIC PERCEPTION, SHOULD WE EXPECT EXPERIENCES TO BE PROBABILISTIC?}

The sensory systems that produce perceptual experience are noisy, since the same external stimulus causes different brain responses on different occasions. In addition, sensory signals in the brain are ambiguous, in that the same signal can be caused by different states of the environment. Keeping track of these two forms of uncertainty could benefit the perceiver, and there is evidence that the perceptual system takes account of both noise and ambiguity. ${ }^{12}$

How does the perceptual system take account of uncertainty? Many models of perception posit computations over probabilistic information, or approximations of such computations, about both sources of uncertainty. Such attributions of probabilistic representations in the perceptual system are made on the basis of behavior predicted by probabilistic models, and in that

\footnotetext{
${ }^{11}$ Another challenge concerns interactions between phenomenal features of experience. The models discussed by Denison et al. are designed to explain perceptual decisions about individual features such as orientation or the direction of motion. Some aspects of perceptual experience involve multiple features that interact phenomenally, for instance, if the color of a stimulus depends on the colors of the things around it, or the sound of a phoneme depends on its surrounding phonemes, or the speed at which a thing looks to be moving depends on the luminance features. Some cross-modal effects have this structure as well, such as the McGurk effect involving vision and audition. For many phenomenal features of perceptual experience, it may not be possible to leverage models of individual features into predictions about perceptual experience.

${ }^{12}$ For evidence old and new see Helmholtz (1856)/1962), Ernst and Banks (2002), Weiss et al (2002), Trommershäuser, Kording, and Landy (2011), and O'Callaghan, Kveraga, Shine, Adams, and Bar (2017).
} 
sense the behavior is sensitive to probabilistic information. In these cases, there appear to be probabilistic information in perceptual processing and behavior that is sensitive to that information, where the behavior is also possible to rationalize by perceptual experience.

For instance, Walker et al. (2020) gave monkeys a task to decide which of two distributions over orientations a grating belongs to, where the two distributions share a mean but differ in variance. Some orientations belong to both distributions, while others belong only to the distribution with the wider variance. After training the monkeys to classify gratings correctly by color (e.g., a sample was colored red if it belonged to the wide distribution and green if it belonged to the narrow one), the color was removed from the samples, and the monkeys had to decide which distribution samples belonged to without relying on color cues. This is the behavior that turned out to be sensitive to probabilistic information.

The sensitivity showed up on trials where the orientation of the sample belonged to both distributions, and the sample was also shown at low contrast. The low contrast introduced noise in the monkey's measurement of the sample's orientation. As before, the monkeys had to decide which distribution the sample belonged to. Their responses were predicted by a model that combines information taking account of the noise surrounding the measurement of the sample's orientation, with information about each distribution over orientations. In a nutshell, the monkey's behavior matched the behavior that would be produced if they reasoned that, for orientations that lie near the intersection of the two distributions, the noisier a sample is, the more likely it belongs to the narrow distribution, because that distribution contains more orientations close to the measurement shown with noise attached to it.

On this model, the monkeys behave as if they are computing over two kinds of probabilistic information: how likely the sample is to have various orientations, and how likely each of those orientations is to belong to each distribution. Their behavior is sensitive to probabilistic information, and therefore to uncertainty registered by the perceptual system.

Switching from monkeys to humans, Walker et al.'s (2020) results belong to a body of evidence that perceptual systems take account of uncertainty by using probabilistic information, though it remains a major question what sorts of computations are involved. ${ }^{13}$ If that suggestion is correct, it adds to the evidence that some human behavior that we regularly take to be directly responsive to perceptual experience is also sensitive to uncertainty registered and used by the perceptual system.

If perceptual experiences can be probabilistic, then there would be a throughway for probabilistic information to flow from subpersonal perception to consciousness, which could in turn explain behavior that is sensitive to probabilistic information. This picture has no need for transitions between non-probabilistic perceptual experiences and probabilistic information.

This observation suggests the line of thought that I will criticize in Section 4. Suppose that perceptual experience has no way to express any probabilistic nuance, because it cannot be a state of uncertainty. If behavior that we regularly assume to be guided by perceptual experience is also sensitive to probabilistic information in subpersonal perceptual processing, it becomes pertinent to ask whether experience is actually epiphenomenal when it comes to explaining behavior sensitive to probabilistic information. If the behavior is explained by the probabilistic information that is left out of perceptual experience, what explanatory role, if any, is left for the experience to play?

${ }^{13}$ For examples of the vast discussion of this issue, see Knill and Richards (1996), Shi, Griffiths, Feldman, and Sanborn (2010), Griffiths, Chater, Kemp, Perfors, and Tenenbaum (2010), Ma (2012), Icard (2016), and Rahnev (2017). 
Some defenses of the idea that probabilistic perception reaches all the way to experience are framed around the supposed choice between probabilistic experiences and epiphenomenal ones. As noted earlier, Morrison (2019) argues explicitly from the implausibility of epiphenomenal experiences, together with experimental support from Walker et al., to the idea that perceptual systems take account of uncertainty by using probabilistic information. We met this line of thought earlier, in the introduction. It says that if perceptual experience explains behavior, and some of that behavior is sensitive to probabilistic information, then perceptual experience must be a vehicle for probabilistic information to which the behavior is sensitive.

Munton (2016) follows a similar line of thought when she defends probabilistic experiences. She appeals to the explanatory role of such experiences in forming and justifying credences, which are probabilistically structured degrees of confidence, rather than behavior, but the basic line of thought is the same. The idea that experiences are probabilistic is a natural hypothesis, given the assumptions that there are behaviors sensitive to probabilistic information that perceptual experiences seem to help explain. Munton and Morrison use this line of thought in defense of probabilistic experiences, but it could also be marshalled in defense of epiphenomenal ones. When we ask whether non-probabilistic experiences could explain behavior that is sensitive to probabilistic information, what kind of explanatory role is at issue?

We can distinguish between a merely causal role for experiences in explaining behavior, and a role that makes the behavior intelligible from the subject's own perspective, which I will call a firstperson rationalizing role for experience. A different way for an experience to be epiphenomenal relative to a behavior is for it to fail to play any causal role in producing the behavior.

The most robust explanatory role for experience would combine both causal and first-person rationalization roles. I will argue that perceptual experiences can provide first-person rationalizations of probabilistic behavior, even if the experiences are not probabilistic. I will leave aside what causal roles experiences actually play relative to such behavior. ${ }^{14}$ I focus on the first-person rationalization role because the methods available to philosophy are well-suited for finding out whether experience plays that kind of role, whereas discovering its causal role can require experimentation. A related reason to focus on the first-person rationalization role is that it is central to the commonsense assumptions about experiences that the line of thought favoring probabilistic experiences relies on.

If my conclusions are correct, then experiences are available as first-person rationalizations of behavior sensitive to probabilistic information, regardless of their causal role in producing that behavior.

\section{4 | ARE PROBABILISTIC EXPERIENCES NEEDED TO EXPLAIN PROBABILISTIC BEHAVIOR?}

Suppose a subject's perceptual experiences have no way to reflect some of the information to which their behavior is sensitive. Would that force the experiences to be epiphenomenal with respect to that behavior?

No. Perceptual experiences can participate in a division of explanatory labor with unconscious perceptual information, postperceptual information, or both.

\footnotetext{
${ }^{14}$ Compare the controversies surrounding Libet's conclusions about free will, which seem to contradict the commonsense assumption the states our brains were in before we decide to act do not prevent our decisions from being free (Bayne, 2004; Libet, Wright, \& Gleason, 1982; Wegner, 2002).
} 
For instance, suppose experience has no way to reflect any probabilistic nuance, but that it can present a subject with multiple incompatible possibilities at once, thereby making them salient to the perceiver. And suppose that postperceptually, the subject attaches probabilistic weights to each option. Here, one could read off from experience the hypotheses to which probabilistic weights are assigned, even if one could not read off the weights that are assigned to each of them. One would not be reasoning in the dark, if one assigned probabilistic weights to multiple hypotheses made salient by one's experience, such as the hypothesis that a darting butterfly is on a leftward trajectory and the hypothesis that it is on a rightward trajectory.

A different way for experiences to rationalize behavior in light of the contribution from other non-experiential states is illustrated by behavior that is guided by the dorsal stream, such as the visually impaired subject DF's ability to post a card through a slot. ${ }^{15}$ DF's visual experience is degraded so much that she cannot see how the slot is oriented. Yet she can post a card through it, successfully positioning her hand to match the card's orientation with the orientation of the slot. DF's sensory-motor success is apparently not guided by any visual experience of orientation, but instead by orientation information that bypasses it, while remaining available for her to use. Her visual system processes information about the slot's orientation, but her experience does not inherit that information.

If the orientation information DF uses never reaches consciousness, that fact does not make her visual experience irrelevant to explaining how she posts the card through the slot. If DF's visual experience indicates the direction in which the slotted surface is located, then it will not be entirely epiphenomenal. By contrast, if her visual experience is too degraded to provide any information that DF could use to guide her card-posting behavior, then it will be. The crucial factor in determining whether experience is epiphenomenal is what information other than orientation the experience conveys, not whether any unconscious information guides behavior.

A special case of unconscious information is unconscious probabilistic information. Perceptual experience can retain a first-person rationalizing role with respect to behavior, even when it joins forces with unconscious probabilistic information to which the behavior is sensitive. Here are three examples.

First, experiences could present a point value estimate of a feature, such as a degree of orientation or a distance, and then any uncertainty surrounding that point value could be encoded in the role of experiences in subsequent reasoning that uses probabilistic information. One could not recover probabilistic information from the experience alone. However, one could recover it jointly from the experience, which phenomenally presents a point estimate of orientation, and other perceptual states, which encode the variance surrounding that point estimate. A proposal like this is made by Shea and Frith (2019). ${ }^{16}$

Second, suppose an experience has the phenomenal content $p$, with which it phenomenally represents an orientation. Since $p$ is a phenomenal content, any two experiences with that orientation content $p$ will be the same with respect to the phenomenal character that $p$ helps characterize. And suppose the same experience also non-phenomenally represents a hedge on that orientation. For instance, the experience has non-phenomenal probabilistic content $.7 p$ if .7 measures how strongly you are disposed to rely on this experience that $p$ in subsequent reasoning, what confidence reports you are disposed to make, and whether you are disposed to look

\footnotetext{
${ }^{15}$ Goodale and Milner (1992). Like many participants in experiments, DF is known by her initials. See Ganel and Goodale (2019) for discussion of the status of DF's experience.

${ }^{16}$ For Shea and Frith (2019), the metacognitive representation of the variance is a phenomenally conscious and part of perception, though its contents do not co-vary with phenomenal character.
} 
again before you act on the information. ${ }^{17}$ However, these elements could be accompanied by a middling amount of felt fluency in producing a report on the orientation, or a feeling that the answer is on the tip of the tongue, or a general feeling of uncertainty. ${ }^{18}$ Here, probabilistic behavior is rationalized from a first-person perspective by phenomenal features that only contingently accompany (non-phenomenal) probabilistic contents: The phenomenal content $p$ of perceptual experience, and the possibly non-perceptual feelings of fluency, and so forth. The perceptual experience can still contribute to first-person rationalizations of behavior.

Finally, Vance (2020) argues that degrees of blur in experience rise and fall with degrees of precision registered in processes that produce it, and that blur is a manner of representing external-world contents. If blur could make it rational from a subject's point of view to become uncertain about how far away something is or exact orientation, then we have another potential division of labor in explaining behavior that is sensitive to probabilistic information. Blur would provide the first-personal rationalization for uncertainty, while unconscious probabilistic information explains more fine-grained patterns of behavior (an example is coming shortly). ${ }^{19}$

These examples show that experiences can contribute to first-personal rationalizing explanations of behavior, even when the behavior is sensitive to unconscious probabilistic information.

My conclusion so far is an instance of a more general claim: Building uncertainty into experience is not needed to explain why a subject ends up uncertain about something she consciously perceives. Suppose you want to know the age of a person you are seeing. The wrinkles on their hands suggest they are over 40, while their hair color suggests they are under 40. So you have two competing suggestions, which together leave you uncertain as to whether this person is over 40. Yet you need not be uncertain about how wrinkled or grey you find their hands or hair. So it is not true in general that a mental state that explains why a subject ends up uncertain has to be a state of uncertainty of its own.

A final consideration against expecting continuity of probabilistic information from perceptual processing via experience to behavior becomes visible if we look backward from perceptual experience to subpersonal processes that produce it. Suppose your visual system stores information of the form: "under conditions C, it's $80 \%$ likely that the important, valuable interesting stimuli are to the left," and as a result, $80 \%$ of the time when you are in conditions C, you look to the left. Then, your pattern of sensory behavior is reflecting uncertainty registered by your visual system. And since this behavior helps determine what you perceptually experience, a pattern of experience is reflecting this uncertainty as well.

This kind of propensity to look to the left is a disposition, it can come in different strengths, and those differences could be measured by probability (Icard, 2016). So here is a case where probabilistic information upstream of experience leaves its mark on the pattern of experiences you have, namely the experiences of whatever is on your left. However, for all the pattern shows, you could not read off any probabilistic information from the phenomenal character of experience, even if you could read off from the frequency of such experiences. In this way, sensory behaviors that are calibrated with probabilistic information would provide a way for nonprobabilistic experiences to reflect uncertainty, without being a state of uncertainty.

\footnotetext{
${ }^{17}$ Since these things could in principle come apart, I am idealizing in supposing that there is a single dimension of strength that the probabilities can measure here. Exactly what dimensions of perception probabilistic can usefully measure is a central question in assessing the general idea that perception is probabilistic. I return to this question in Section 6. See also Pohl (2019).

${ }^{18}$ These proposals come from Shea (personal communication).

${ }^{19}$ For blur to provide a first-person rationalization of behavior, must it be an intrinsic phenomenal hedge-marker? No. This point is discussed in Section 5.1.
} 
All of these observations count against the line of thought that if perceptual processing is probabilistic, and if behavior is sensitive to any of the probabilistic information processed, then we should expect perceptual experience to be probabilistic, so that a special explanation is needed of why it is not probabilistic, if that is what we find. ${ }^{20}$ If there is no reason to expect experiences to be probabilistic just because perceptual processing is, then no special explanation is needed. What would be needed instead, arguably, is an account of how experiences help explain probabilistically sensitive behavior if they are not probabilistic.

I have given reasons to doubt that the relationships between probabilistic perceptual processing and perceptual experience and behavior support probabilistic experiences. I now consider the more fundamental question whether any experiences can intrinsically be states of uncertainty. I will argue that some experiences may well be states of uncertainty-contrary to the standard picture of perceptual phenomenology described in Section 2. In addition to challenging a longstanding assumption about perceptual consciousness, this result gives us some leverage on assessing whether any experiences are probabilistic.

\section{5 | THE CHALLENGE FROM WOBBLY TRAJECTORIES}

The standard picture is that perceptual phenomenology, as distinct from phenomenal imagination and phenomenal memory purports to present subjects with a unique way in which external things are actually present at the moment of perception (Figure 1). This picture is compatible with phenomenal features that mark a hedge by the roles they play in the mind, but only when those roles are only contingently played by the experience.

For instance, earlier we discussed probabilistic information taken account of by perceptual processing that remains unconscious. Some philosophers, including Shea (2018), develop theories of content according to which some such probabilistic information is represented in perceptual experience, in the form of non-phenomenal content (by definition, having such content is a representational feature that does not supervene on phenomenal character). You could hold constant the phenomenal character of the experience and vary whether the subject is related to the environment in ways that would establish the kind of representation relation that is definitive of content as Shea construes it.

The standard picture is also compatible with phenomenal features that only contingently cue uncertainty. For instance, if you learn to associate a high pitch with uncertainty about orientation of a line that you see or touch, nothing intrinsic to the pitch tells you about orientation or how uncertain you should be about it. Or if your experience leaves you disposed to make confidence reports partly based on it, that disposition, too, may be only contingently associated with an experience. For instance, you might experience a line oriented at $+5^{\circ}$, but in one condition a subject might be disposed to report relatively less confidence that it has that orientation, and in another condition one might be disposed to report relatively more, even though the phenomenal character is the same both times.

In these examples, the roles of experience that mark the hedges are: a disposition to produce confidence reports in response to experience; and being related to the environment in a way that establishes the experience as a representation of probabilistic information. The standard picture is compatible with experiences that play either of these roles.

\footnotetext{
${ }^{20} \mathrm{An}$ evolutionary explanation is offered by Clark (2018), who suggests that probabilistic experiences would be worse at guiding decisions than nonprobabilistic ones and therefore would not be selected.
} 
By contrast, the standard picture is incompatible with phenomenal features that in their own nature (intrinsically) qualify information about the external world. What kinds of phenomenal features could constitute such a hedge?

A first kind would be a meta-cognitive layer of representation about the status of other contents of experience. For instance, an experience might present a line as oriented $10^{\circ}$ to the left, and then include another representation that the line might be a bit more or less than $10^{\circ}$.

The central challenge is to characterize the phenomenal hedge, without relying on roles that only contingently accompany the phenomenal character, or on roles that establish nonphenomenal probabilistic contents, or on feelings of uncertainty formed in response to perceptual experience. It is a difficult challenge because perceptual experience purports to tell us how things are in in our immediate environment, whereas uncertainty surrounding perception is about our relationship to the information about those circumstances. Is there any hedge intrinsic to perceptual consciousness?

A natural suggestion is that in the visual case, blur is an intrinsic phenomenal hedgemarker. Suppose that Vance (2020) is correct that degrees of blur rise and fall with degrees of precision. If there is an informational throughway for probabilistic information of the sort under discussion here, then blur should be a phenomenal feature that conveys probabilistic information. A. D. Smith may have been suggesting as much when he wrote "the animal response to blur is uncertainty" (Smith, 2008). More exactly, blur would be an intrinsic phenomenal hedge-marker if it were non-contingently related to a perceptual mode of middling-tolow proto-confidence.

However, what are proto-confidences? The face-value argument may help operationalize them. We can ask: Guided only by experience of a feature, without relying on background beliefs or conditioned habits (such as associating high pitch with uncertainty about orientation), would it make sense from a first-person perspective to end up uncertain about that feature?

We can use this operationalization to assess whether blur is a phenomenal hedge-marker. To develop it into a proposal about blur that is exact enough to assess, it can be refined:

For any feature $\mathrm{F}$ presented in experience, if you blurrily experience something as $\mathrm{F}$, then going just by experience, it would make sense from a first-person perspective to become uncertain about whether the thing is $\mathrm{F}$.

A complementary idea is that degrees of blur rise and fall with how uncertain it would make sense to be about whether the thing really is F, when guided just by your experience. Analogous proposal could be formulated for low contrast. ${ }^{21}$

These proposals seem to be false. For many features F, some amounts of blur need not leave person any less uncertain that they would be if they were experiencing something as F clearly instead of blurrily. Blurred red can still appear red, a blurred human form still appear plainly to be a human form, and blurred things moving toward me can still plainly appear to be moving toward me. Increasing blur need not leave me less certain that the form is human, and decreasing it need not increase my certainty.

Smith's idea that blur per se is a phenomenal hedge-marker may arise from mistaking it for a different point: that compared to clear vision, blurred vision changes the information one can get from a scene by reducing spatial frequencies, presenting obstacles to the flow of some kinds

\footnotetext{
${ }^{21}$ For example, "For any feature F presented in experience, if you experience $\mathrm{F}$ at low contrast, it would make sense from a first-person perspective to become uncertain about whether the thing is F."
} 


\begin{tabular}{|l|l|l|l|l|}
\hline & actual & actual & merely & future \\
\hline present & past & possible & \\
(not actual) & \\
\hline perception & $X$ & & & \\
\hline memory & & $X$ & & \\
\hline imagination & & & $X$ & $(X)$ \\
\hline
\end{tabular}

FIGURE 1 A standard view of phenomenal invariances

of information. It can be difficult to read with vision so blurred that the lines distinguishing the letters from one another cannot easily be made out. However, what is at issue here is whether blur per se is a hedge marker, operationalized as rationally generating uncertainty if one is not relying on background information. The fact that it does not always play that role shows that nothing internal to blur makes it constitute a hedge on external-world content.

Similar points hold for low contrast. Backlit cubes can look just as cubical as fully lit ones, and if I end up confident that it is a cube, I need not be relying on information independent from my visual experience.

These considerations reinforce the challenge of finding a pair of phenomenal aspects of experience, where one aspect qualifies the status of what is presented in the other aspect. A way to meet this challenge is to find cases in which experiences present multiple incompatible possibilities at once, by making such possibilities salient in the subject's perceptual consciousness. We can say that experiences structured in this way are multiplex. ${ }^{22}$ The structure of multiplex experiences, I will argue, provides a more promising candidate for intrinsic phenomenal hedge-markers than blur.

\section{1 | Multiplex experiences}

Being multiplex per se does not suffice for making experiences into a state of uncertainty (or therefore into an intrinsic phenomenal hedge-marker), because some multiplex experiences present a single object as having incompatible features, such as the magic fork (Figure 2) which looks to have two prongs at one end, and at the other end it looks to have three prongs. Just as (unfortunately) there need be no hedging if someone asserts a contradiction, in principle, it seems, one could experience an impossible figure without one's experience hedging on whether the item really has all the features it appears to have. The Escher staircase is visually compelling to look at because, amazingly, it seems to keep going up even while forming a circuit.

\footnotetext{
${ }^{22}$ The possibilities are made salient in the minimal sense that they are present to the subject's mind. The experience has contents that characterize multiple scenarios, making those scenarios present to the mind as distinct ways the world could be. For a richer notion of salience used to the describe the internal organization of experience into things relatively more peripheral or central to perceptual consciousness, see Dicey-Jennings (2020) and Watzl (2017).
} 
FIGURE 2 The magic fork

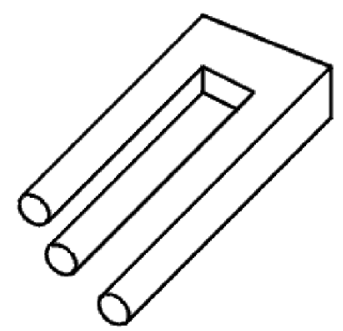

Multiplex experiences will be states of uncertainty if the possibilities made salient are experienced as exclusive. Can perceptual experience make a range of incompatible possibilities salient to the mind, where the possibilities in the range are marked with a hedge indicating that the scenarios presented are ways things might be, but also might not be ${ }^{23}$

A natural candidate for multiplex states of uncertainty comes from the kind of multistable experiences typically caused by binocular rivalry. If you are shown a different stimulus in each eye, such as a house and face, typically your experience will flip between a house-percept and a face-percept. ${ }^{24}$ Because the information from each side of the flip is not easily integrated, it would be rational and normal to remain uncertain of what you are seeing, if you did not know you were getting different stimuli in each eye.

However, what if each experience that you flip between is a distinct experience? Then we will have alternation between non-multiplex experiences, rather than a single multiplex experience presenting different incompatible possibilities.

In this case, individuation is a partly conceptual matter. You could hold constant the stimuli and the phenomenal character, but change only the theoretical decision about whether the subject is undergoing a single dynamic experience or a sequence of separate ones. $^{25}$ So whether multistable experiences are states of uncertainty is somewhat up for grabs. A stronger candidate would be a multiplex experience that presents us with incompatible trajectories over a short duration, but where we have a firmer motivation to treat it a single dynamic experience.

The structure of these cases is analogous to someone who says on Sunday that since Friday they have uninterruptedly been and are continuing on a single career trajectory: to become a sculptor. And then on Monday say that since Friday, they have uninterruptedly been and are continuing on a single career trajectory: to become a lawyer. Such a person's reports could not both be true. Experiences of wobbly trajectories may have this same structure.

\footnotetext{
${ }^{23}$ In case it helps bring this question into focus, consider a nonperceptual analogy. Suppose that while you are wondering whether the sound you hear in the desert is caused by a coyote or a baby, you imagine that it is caused by a witch disguising herself as a baby. Here you are actively holding in your consciousness these three incompatible options—-Сооте, ваву, and шітсH—and none of them are marked in your consciousness as "actual." The соуоте and BABY options are epistemic possibilities for you that pass through your mind while you are in the throes of inquiry, whereas wIтcH is an epistemically impossible far-off (logical) possibility brought to mind by your creative imagination ( $p$ is epistemically possible for a subject $\mathrm{S}$ iff $p$ is possible, for all $\mathrm{S}$ knows). As this case illustrates, imagination and inquiry can make a range of incompatible possibilities of various kinds salient to you, as can mind-wandering, conversation, and reading. The question here is whether perceptual experience can perform the same function that imagination or inquiry can.

${ }^{24}$ In some conditions, there can be fusion (Block, 2018; Gershman, Vul, \& Tenenbaum, 2012; Klink, Boucherie, Denys, Roelfsema, \& Self, 2017).

${ }^{25}$ Lewis (1986) makes a similar proposal about event individuation.
} 


\section{2 | Butterflies and knuckleballs}

It is hard to catch a butterfly as it darts and flutters because none of its movements allow us to predict what its next movement will be. If we watch long enough, for instance for $5 \mathrm{~s}$, we might see its overall direction of movement, but smaller segments of this trajectory would offer competing cues to its overall direction. As in multistable experiences, the temporal segments of the experience present incompatible things. In one microsegment of the experience, the butterfly darts to the left, and the next microsegment it darts to the right. However, unlike multistable experiences that alternate between house and face, here, if we consider the experience encompassing both segments, we have competing cues as to its trajectory.

The same situation on an even smaller time scale can be found in a type of pitch in baseball known as a knuckleball, one of the most unpredictable breaking pitches. Knuckleballs dart and waffle, making it hard to predict the ball's path and hit it. ${ }^{26}$ Since trajectories are plausibly features presented in experience over durations, these experiences present you with multiple incompatible trajectories at once.

At this point, one might object that none of these examples are really experiences of multiple options as incompatible, on the grounds that what is presented in experience is something of the form "Possibly P," such as "it is possible that the ball is on trajectory T1" and "it is possible that it is on T2." And just as you could believe two claims of the form "Possibly P" without contradiction, if you experienced both "possibly $x$ is on trajectory T1" and "possibly $x$ is on trajectory T2," this would not be a case of experiencing multiple incompatible options.

The challenge might seem to be deepened by comparing it to an experience of instability, a disposition of something wobbly to topple over. For instance, imagine a visual experience of a rock balanced precariously on another rock. Here, one is experiencing a property (instability) that the rock actually has. The experience need not be hedging on whether the rock is unstable, or on how unstable it is.

In reply, the experiences described here are hedging on which trajectory is currently unfolding. In granting that the phenomenal contents of the experiences include "Possibly the trajectory is T," the objector grants there is a phenomenal hedge. ${ }^{27}$

The experience of the rock is different. This experience need not be hedging on whether the unstable rock will actually fall. Any uncertainty about that is a response to the perceptual experience of instability. ${ }^{28}$ It would be reasonable to be uncertain whether the rock will fall in response to the experience, but if the rock is balancing without moving at all, there is no ambiguity about its trajectory because it is not on a trajectory.

\footnotetext{
${ }^{26}$ https://en.wikipedia.org/wiki/Knuckleball.

${ }^{27}$ The kind of possibility would have to be a type of epistemic possibility, so that $p$ is possible for $\mathrm{S}$ in this sense just in case: "For all S would be justified in believing on the basis of S's experience alone: $p$ " For instance, if an experience of a dotted card would give you with immediate justification to think the card has nine dots, and would also give you justification to think it has 10 dots, then "Possibly: It has nine dots" would be a content of experience. In her discussion of this example (which is from Silins, 2011). Munton (2016) suggests that experiences hedge in just this way.

${ }^{28}$ Compare McGinn's (1996) claim against dispositionalism about color that "You do not see what would obtain in certain counterfactual situations; you see only what actually obtains. When you see something as red you do not see the counterfactual possibilities that constitute its having a disposition to appear red. Your eyes do not respond to woulds and might have beens" (1996, p. 540). McGinn is moving illicitly from the plain fact that we cannot perceive what is merely possible to the conclusion that we cannot be presented with dispositional properties. It is part of "what actually obtains" that the rock is disposed to topple over (i.e., that is unstable), and plausibly this dispositional property can be presented in experience.
} 
If no experiences were states of uncertainty, then there would be no probabilistic experiences either. If some experiences of wobbly trajectories are states of uncertainty, then the standard view would need to drop the requirement that perceptual experiences present a unique way that things actually are. An adjusted version of the distinction might hold that perceptual experiences purport to present the subject with ways things actually are at the time of the experience, allowing that multiplex experiences concern candidates for how things actually are at that time, rather than ways things were in the past or could be at some other time or in some other world.

My cases for wobbly experiences as states of uncertainty do not rule out all possibly alternatives. In the case of knuckleballs, one might try to argue that the experience presents shifts in the ball's direction, without specifying which directions these are. This analysis would not create any need to adjust the standard view at all. What is important in this context is that if some experiences may well be states of uncertainty, and if any are, then that result opens the question whether this experiential uncertainty is ever probabilistically structured or otherwise measurable by probability.

From here, I am going to grant for the sake of argument that some experiences are states of uncertainty and argue that none of them are probabilistically structured or measurable. Readers who are think that no experiences are states of uncertainty will have reached the same conclusion already.

\section{I ARE EXPERIENCES PROBABILISTIC? THE PROTO-CONFIDENCE ANALYSIS}

So far, I have argued against the standard picture of perceptual phenomenology, according to which all perceptual experiences present things as actually occurring concurrently with the perception. I made the case that some experiences of conflicting trajectories falsify this universal claim.

A special case of an experience presenting the subject with multiple incompatible possibilities would be that it presents possibilities as weighted. Here, the phenomenal hedge would also be weighted in a way that forms a probability distribution. This picture can be developed into what I will call the proto-confidence analysis of experience.

Just as the standard picture makes a universal claim about all experiences, the protoconfidence analysis makes equally far-reaching universal claims. It is an analysis of how phenomenal character is structured. According to the version I will consider here, it is structured by a set of relations I will call "perceptual seeming relations."

A relation to contents is a perceptual seeming relation, if it is a relation to contents by virtue of which you are phenomenally perceptually presented with the things the contents characterize, and if the relation is distinct from belief, judgment, or anything else doxastic. For instance, if you are phenomenally perceptually presented with a person coming to greet you and the same person coming to greet someone else, then on this analysis, you would stand in two perceptual seeming relations, each one to a content characterizing each of the possibilities. On this analysis, experience can make salient multiple incompatible possibilities at once.

A further step assigns weights to them that are measurable by probabilities. This step develops two ideas: that there are intrinsic phenomenal hedge-markers in the form of degrees of perceptual seeming relations, and that these hedge-markers are probabilistically structured. 
I will call any degreed perceptual seeming relations measurable by probabilities "protoconfidence relations." Just as there are many credence relations, there are many protoconfidence relations. In this framework, there could be such a thing as its perceptually seeming to degree .7 that a sound is made by a coyote, and its simultaneously perceptually seeming to degree .3 that the sound is made by a human baby. The witch hypothesis from our earlier example would not show up in proto-confidences because it is made salient by imagining, not by perceptual experience. ${ }^{29}$

Proto-confidences would be experiences built out of proto-confidence relations. If experiences are proto-confidences, then they have features that merit using probabilistic formalism to describe them. Formal structures such as probability are apt for analyzing psychological states only when those states independently have the structure that the formalism helps describe. The conceit of the proto-confidence analysis is that experiences have such features. There is a dimension of phenomenal character of perceptual experience that comes in degrees measurable by probability. Since nothing in the formalism itself identifies what thing in the mind the formalism measures, that task is left to some other type of analysis, such as phenomenology for perceptual experience, cognitive modeling for perceptual processing, or philosophy for credences.

It is worth noting that in their critical survey of the history of attempts to analyze the type of confidence measured by probability, Ericsson and Hajek (2007) conclude that none of those attempts succeed, and suggest that credences are best taken as a psychological primitive. Their discussion highlights the challenge of finding person-level psychological underpinnings for probability to measure. If Ericsson and Hajek's criticisms of all extant attempts to operationalize credences are correct, it suggests that the best reasons to think the mind is probabilistically measurable will come from other things that can be explained on that assumption.

An explanation of that sort is exactly what Munton and Morrison offer for perceptual experience. I have focused on their strategy for defending proto-confidences because it says protoconfidences are needed to explain probabilistic behavior. In Section 4, I criticized this strategy. Munton also makes the case that proto-confidences can explain the formation of rational credences. I return to this point shortly.

I now to turn from a strategy used to defend proto-confidences to the proto-confidence analysis itself. The key question is whether there is a phenomenal dimension to experience that can be measured by probabilities. By using the same formalism for a different purpose, the protoconfidence analysis commits to the idea that experiences are similar enough to credences to merit probabilistic analysis, but different enough from credences to be perceptual precursors of credences, rather than credences under another name.

Morrison (2015) proposes the proto-confidence analysis as an account of invariant phenomenal features of perceptual experience. When introducing the view (which he calls "perceptual confidence"), he writes:

Perceptual confidence is ... about how your experiences present objects and properties ... [it] extends an earlier shift in our thinking. In the 1960 s ... many philosophers attributed contents to beliefs but not to perceptual experiences. In the 1980s many started attributing contents to experiences ... because they became convinced

\footnotetext{
${ }^{29}$ In illustrating the proto-confidence analysis, I have used an example involving the sophisticated property of being a coyote to maintain continuity with earlier examples. However, the proto-confidence analysis does not depend on the idea that such sophisticated properties could not be representable in perceptual experience.
} 
that experiences are belief-like in many ways ... Jumping to the present, many contemporary philosophers attribute degrees of confidence to beliefs but not to experiences ... According to perceptual confidence, experiences are belief-like in yet another way: they can assign more or less confidence ... One could selectively endorse Perceptual Confidence in some cases but not others. But it most naturally applies to all or none. (Morrison, 2015, p. 19). ${ }^{30}$

The proto-confidence analysis modifies the set of contrasts posited by the standard view. It says that perceptual experience can identify a range of scenarios and presents each of them as a way that things might actually or might not be, instead of presenting a single way things are now.

As noted a few paragraphs back, Munton's (2016) defense of the proto-confidence analysis draws on epistemological considerations. She writes:

What properties must our experience have if it is to account for ... fluctuations in epistemic power, even across experiences that share epistemically relevant contents? I propose that we should understand the relevant difference in such cases as a change in the certainty or confidence with which the visual state presents its content. (Munton, 2016, p. 302).

Munton makes explicit that differences in proto-confidences are also phenomenal differences:

The phenomenal character of a visual experience can change incrementally. How things look to you develops gradually as you move, as the scene you are looking at evolves, or as environmental conditions change. Those changes in phenomenal appearance have epistemic upshots: your epistemic position also changes very gradually, in line with them. (Munton, 2016, p. 302).

The contents of experience can stay the same, she suggests, as epistemic power changes, which is what motivates the idea that relations to contents make a phenomenal difference as well. As she puts it: "Note that at some level the contents of Rey's and Elmer's experiences may remain the same, even as their phenomenal character changes in a way that is epistemically relevant" (p. 303).

Munton says one's epistemic position "changes in line with" phenomenal changes, which suggests that if you are proto-confident to degree .7 that it is a tree, .7 measures two things that rise and fall together: something phenomenal, and something epistemic. The phenomenal thing is a degreed dimension of perceptual seeming, and the epistemic thing could be a degree of justification for believing that it is a tree, or a level of confidence that it would be rational to have, such as credence .7 in "It's a tree."

Munton's treatment of the tree example can suggest that a subject's standing in a single proto-confidence relation to a content, making the analysis analogous to a point estimate rather than a distribution. Multiplex experiences are also easily analyzed with degreed perceptual seeming relations, given Munton's assumption that proto-confidences rise and fall with

\footnotetext{
${ }^{30} \mathrm{He}$ adds: "Perceptual Confidence best explains what happens when we completely trust our experience ... when you completely trust an experience you endorse the way it presents objects" (p. 27).
} 
epistemic power. If in looking at a cloth in dim light, it would be rational to form credences .4 that it is blue, .3 that it is green and .3 that it is purple, Munton's analysis can be expressed by positing three corresponding proto-confidence relations that the subject's experience consists in. ${ }^{31}$

The proto-confidence analysis can be developed into either type of theory. However, relative to the goal of showing that perceptual experiences are intrinsic phenomenal hedge-markers, the distribution version is more promising. Compared to proto-confidence analyses of multiplex experiences, the point-estimate version leaves it less obvious what the phenomenal hedgemarker is. The putative fact that having an experience makes it rational to form one or another credence could be explained on the standard view as well, as we saw in Section 4. For instance, the experience of seeing a tree in fog that occludes the leafy part but not the trunk might leave it open whether it is a tree or a telephone pole. Here, the features of experience that explain uncertainty about what one is seeing could on the face of it be explained without any phenomenal hedging.

The point-value version of the proto-confidence analysis needs to specify which phenomenal features constitute a hedge-marker. We saw in Section 3 that there may be non-phenomenal roles or representational features of experience that probabilities could measure, and something like a point valued version of the proto-confidence analysis could describe experiences in this way. However, those theories do not posit any intrinsic phenomenal hedge-markers.

Given Munton's and Morrison's goal to make predictions about all perceptual experiences, there is some pressure on their proto-confidence analysis to posit multiplex experiences. We are then left with a question: Do multiplex experiences lend themselves to probabilistic analysis?

I argue next that no experiences lend themselves to probabilistic analysis, and therefore multiplex experiences do not either.

\section{1 | The anchor problem and superfluous formalism}

The proto-confidence analysis proposes that all experiences are structured by degreed perceptual seeming relations. Let us suppose for the sake of argument that it provides an illuminating analysis of some multiplex experiences, such as conflicting trajectories or multistable experiences. Why should these types of cases anchor a theory of the structure of all perceptual experiences? We would want some reason to think that these cases are the ones from which we should generalize. Yet there does not seem to be anything especially central or fundamental about these cases. Not every perceptual experience is an experience of a trajectory, let alone ambiguous trajectories, and hardly any experiences are multistable. This objection is the anchor problem for proto-confidences.

Even if we set aside the anchor problem, the probabilistic formalism does not seem to be tracking anything beyond what we can already describe without it. By invoking probability, the proto-confidence analysis needs experiences to have just the right set of features. There has to be a dimension of perceptual seeming that comes in degrees in a way that probability can measure, this dimension needs to have an upper bound, and all experiences must be locatable in a linear order along this dimension, so that any two perceptual experiences can be compared.

Both the upper bound nor comparability seem more likely artifacts of a formalism than features of phenomena that would make that formalism an apt tool of analysis. Regarding the

\footnotetext{
${ }^{31}$ Compare her example of Silins's dotted card example, and Morrison's (2015) discussion of Jeffrey's cloth example.
} 
upper bound: What would an example be of maximal perceptual seeming? (Is a very vivid cat phenomenally maximal?).

In the case of credences, it is possible to define every credence in terms of credence 1 . If credence 1 is complete certainty, the other credences are progressively less than complete certainty, and we have a psychological underpinning for the formalism. ${ }^{32}$ By contrast, in the case of perceptual seeming, if we have no clear grasp of what maximal perceptual seeming would be, then that fact should leave us dubious that the other values of perceptual seeing are welldefined.

A proponent of proto-confidences might reply to the upper-bound objection by saying that there is no upper bound of proto-confidence. ${ }^{33}$ However, this move introduces new challenges to finding a psychological underpinning of proto-confidence. First, if there is no perceptual analog to complete confidence, it becomes obscure which proto-confidence value should be assigned to epistemically unproblematic perceptual experiences such as seeing one's hand while reaching for a cup. What makes it less than the maximum? Without a clear definition of the phenomenal dimension that is getting measured, the adjusted formalism with no upper bound is not any better motivated than the original formalism.

Regarding comparability, if the proto-confidence analysis is true, then there has to be a fact of the matter about which if either of the perceptual seemings that result from seeing a backlit cat and the Idesawa spiky sphere (Figure 3) are stronger. How would we locate a bang or odor in relation to them? The proto-confidence analysis forces questions on us that do not obviously have answers.

A proponent of proto-confidences could address this objection by denying that there is an upper bound, or by invoking "fuzzy" credences, or both. At that point, however, the motivation that comes from the line of thought emphasizing the continuity of probabilistic information in perceptual processing gets lost. In the Walker et al. study, there is no indication that the probabilistic information guiding behavior is in the form of fuzzy intervals.

FIGURE 3 Idesawa spiky sphere

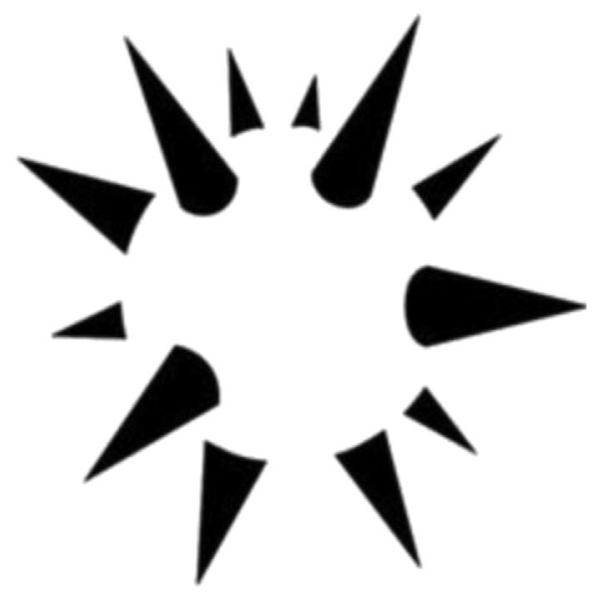

\footnotetext{
${ }^{32}$ Another option is to fix the psychological credence .5 in $\mathrm{p}$ as a type of indifference between $p$ and not- $p$, and then define the other credences as departures in degrees of asymmetry between distributions of confidence in $p$ and not- $p$. The main point is that for the formalism to be useful and apt for analyzing a set of psychological states, there has to be a psychological phenomenon that it helps to make exact.

${ }^{33}$ This suggestion has been made by Munton (personal communication).
} 
This observation points to a further challenge in finding a phenomenal subject-matter for probability that jibes with the motivation from continuity that I have been discussing in this paper. The Walker et al. study is one of the few studies that link probabilistic information to behavior. In other cases of perceiving orientation, even if van Bergen, Ma, Pratt, and Jehee (2015) are correct that probabilistic information is used by perceptual processing of orientation, only the "winning" hypothesis about orientation shows up in perceptual experience. ${ }^{34}$ The phenomenal degrees posited by the proto-confidence analysis then have no role to play. And as I argued in Section 4, non-probabilistic experiences can combine with probabilistic information in the rest of perception to explain behavior.

In sum, probabilistic formalism may be useful for measuring contingent roles of experience, but this use of it is entirely compatible with denying the proto-confidence analysis. That kind of role would not have the momentous consequences for the structure of experience that Morrison and Munton advertise.

\section{7 | CONCLUSION}

When Frank Ramsey first proposed that our levels of confidence can be measured by probabilities, he took confidence, a phenomenon that everyone can recognize, and proposed that probability can help us understand further features it has, at least in ideally rational minds. ${ }^{35}$ There is no doubt about the existence of the subject-matter that the probabilistic formalism was meant to help illuminate.

I have argued that there is no phenomenal dimension to perceptual experience that gives proto-confidences any analogous subject-matter. While probabilities may help us describe some dispositions and roles related to perception, there appears to be nothing phenomenal for probabilities to help us analyze. Perceptual representations of point-estimates may be accompanied by blur or feelings of uncertainty, but these phenomenal feelings are not plausibly measurable by probabilities. The proto-confidence analysis seems to lack a phenomenal subject-matter.

What is the deepest reason why perceptual experiences are not probabilistic? I suspect it is that the core phenomenal features of perceptual experience are to tell us how things are in in our immediate environment, whereas uncertainty surrounding perception is about our relationship to the information about those circumstances. Perceptual experience feels like a tribunal against which we can compare hypotheses about perceptible things. For many reasons, perceptual experience cannot always straightforwardly play that role. ${ }^{36}$ However, basic questions

\footnotetext{
${ }^{34}$ A point emphasized by Clark (2018), Block (2018), and Gross (2020).

${ }^{35}$ Ramsey (1926)/1931), "Truth and Probability." As Ramsey recognized when he founded formal epistemology, the kind of confidence that has the best chance of being analyzed probabilistically are dispositional states of mind, rather than mental occurrences like feelings: "We have therefore to find a process of addition for degrees of belief ... to determine a numerical scale. Such is our problem; how are we to solve it? There are, I think, two ways ... We can, in the first place, suppose that the degree of a belief is something perceptible by its owner; for instance, that beliefs differ in the intensity of a feeling by which they are accompanied ... This view would be very inconvenient, for it is not easy to ascribe numbers to the intensities of feelings; but apart from this it seems to me observably false, for the beliefs which we hold most strongly are often accompanied by practically no feeling at all; no one feels strongly about things he takes for granted. We are driven therefore to the second supposition that the degree of a belief is a causal property of it, which we can express vaguely as the extent to which we are prepared to act on it" (p. 169).

${ }^{36}$ I discuss these reasons in Siegel (2017). See also Christensen (1992), Weisberg (2009), Sturgeon (2020).
} 
about our surroundings, such as (as Austin asked) whether the pig is in the pigpen, normally seem to be questions that perception can settle (Austin, 1962).

If this conclusion is correct, then contrary to premise (P1) of the face-value arguments, one cannot form credences by taking experiences at face-value, simply importing a probabilistic structure of experience to the doxastic level, changing only the nature of the relations one bears to the contents, contra Moss (2019). Credences would have to be formed other ways, such as forming them by inference, or by adjusting your mind to match (what you take to be) the objective chances.

Perception science can tell us much about which behavior is sensitive to probabilistic information. However, the conclusions here suggest that phenomenological considerations may be better than perception science is at assessing whether experiences are phenomenally probabilistic. If my conclusions are correct, models from perception science may be mapped on to states of uncertainty, but not on to any states that are phenomenally probabilistic.

\section{ACKNOWLEDGEMENTS}

I am grateful for a wealth of opportunities to discuss the relationships between probability and perception, including invitations to give the presidential address of the Association for the Scientific Study of Consciousness meetings, the Mind \& Language lecture at the European Society for Philosophy and Psychology, the Royce Lectures at Brown, and the Kripke lecture at CUNY; to speak at conferences at York, Helsinki, Cambridge, and Bochum and give colloquium talks at Carelton University, Glasgow, and Georgia State. I benefitted from discussions on all of these occasions. Thanks to Maria Lasonen-Aarnio, Ned Block, Alex Byrne, Géraldine Carranante, David Chalmers, Rachel Denison, Anya Farrenikova, Jane Friedman, Sam Gershman, E.J. Green, Gabe Greenberg, Steven Gross, Jakob Hohwy, Thomas Icard, Geoffrey Lee, John Morrison, Jessie Munton, Nico Orlandi, Adam Pautz, Christopher Peacocke, Ian Philipps, Stephan Pohl, Katia Samoilova, Miriam Schoenfeld, Nicholas Shea, Maja Spener, Scott Sturgeon, Neil van Leeuwen, Jonna Vance, Fillippo Vindrola, Ege Yumusak, and many others for comments and discussion.

\section{ORCID}

\section{Susanna Siegel (D) https://orcid.org/0000-0001-5554-7677}

\section{REFERENCES}

Austin, J. L. (1962). In G. J. Warnock (Ed.), Sense and sensibilia. Oxford: Oxford University Press.

Bayne, T. (2004). Phenomenology and the feeling of doing: Wegner on the conscious will. In S. Pockett (Ed.), Does consciousness cause behaviour? Cambridge, MA: MIT Press.

Beck, J. (2019). On perceptual confidence and "completely trusting your experience". Analytic Philosophy, 61(2), $174-188$.

Block, N. (1996). Mental paint and mental latex. Philosophical Issues, 7, 19-49.

Block, N. (2018). If perception is probabilistic, why does it not seem probabilistic? Philosophical Transactions of the Royal Society of London B: Biological Sciences, 373(1755), 20170341. https://doi.org/10.1098/rstb.2017.03

Cheng, T. (2018). Post-perceptual confidence and supervaluative matching profile. Inquiry, 61, 1-29. https://doi. org/10.1080/0020174X.2018.1562370

Christensen, D. (1992). Confirmational holism and Bayesian epistemology. Philosophy of Science, 59(4), $540-557$.

Clark, A. (2018). Beyond the Bayesian blur: Predictive processing and the nature of subjective experience. Journal of Consciousness Studies, 25(3-4), 71-87.

Denison, R., Block, N. \& Samaha, J. (2020). What do models of visual perception tell us about visual phenomenology? In F. de Brigard \& W. Sinnot-Armstrong (Eds.), Neuroscience and philosophy. Cambridge, MA: MIT Press. 
Dicey-Jennings, C. (2020). The attending mind. Cambridge: Cambridge University Press.

Ericsson, L. \& Hajek, A. (2007). "What are degrees of belief?" Studia Logica, 86, 183-213. https://doi.org/10. 1007/s11225-007-9059-4

Ernst, M. \& Banks, M. (2002). Humans integrate visual and haptic information in a statistically optimal fashion. Nature, 415, 429-433. https://doi.org/10.1038/415429a

Ganel, T. \& Goodale, M. (2019). Still holding after all these years: An action-perception dissociation in patient DF. Neuropsychologia, 128, 249-254. https://doi.org/10.1016/j.neuropsychologia.2017.09.016

Gershman, S., Vul, E. \& Tenenbaum, J. (2012). Multistability and perceptual inference. Neural Computation, $24,1-24$.

Goodale, M. \& Milner, A. (1992). Separate visual pathways for perception and action. Trends in Neuroscience, 15 (1), 20-25.

Griffiths, T., Chater, N., Kemp, C., Perfors, A. \& Tenenbaum, J. (2010). Probabilistic models of cognition: Exploring representations and inductive biases. Trends in Cognitive Science, 14, 357-364. https://doi.org/10.1016/j. tics.2010.05.004

Gross, S. (2020). Probabilistic representations in perception: Are there any, and what would they be? Mind \& Language, 35, 377-389. https://doi.org/10.1111/mila.12280

Helmholtz, H. (1856/1962). Treatise on physiological optics. New York, NY: Dover Publications.

Hobson, A. (2005). Dreaming: A very short introduction. Oxford: Oxford University Press.

Husserl, E. (2005). Phantasy, image-consciousness, and memory. In R. Bernet (Ed.), Edmund Husserl: Collected works, volume 11. Dordrecht: Springer.

Icard, T. (2016). Subjective probability as propensity sampling. Review of Philosophy and Psychology, 7(4), 863-903. https://doi.org/10.1007/s13164-015-0283

Klink, C., Boucherie, D., Denys, D., Roelfsema, P. \& Self, M. (2017). Interocularly merged face percepts eliminate binocular rivalry. Scientific Reports, 7, 7585. https://doi.org/10.1038/s41598-017-08023-9

Knill, D. \& Richards, W. (Eds.) (1996). Perception as Bayesian inference. Cambridge: Cambridge University Press.

Lau, H. (2019). Consciousness, metacognition, and perceptual reality monitoring (Version 1). PsyArXiv. https:// doi.org/10.31234/osf.io/ckbyf

Lewis, D. K. (1986). Events. In Philosophical papers (Vol. II, pp. 241-269). Oxford: Oxford University Press.

Libet, B., Wright, E. \& Gleason, C. (1982). Readiness potentials preceding unrestricted spontaneous pre-planned voluntary acts. Electroencephalography and Clinical Neurophysiology, 54, 322-325.

Ma, W. (2012). Organizing probabilistic models of perception. Trends in Cognitive Sciences, 16(10), 511-518.

Madary, M. (2012). How would the world look if it looked as if it were encoded as an intertwined set of probability density distributions? Frontiers in Psychology, 3, 419.

Martin, C. B. \& Deutscher, M. (1966). Remembering. Philosophical Review, 75(April), 161-196.

Martin, M. G. F. (2002). The transparency of experience. Mind \& Language, 17(4), 376-425.

Mazzoni, G. \& Memon, A. (2003). Imagination can create false memories. Psychological Science, 14(2), $186-188$.

McGinn, C. (1996). Another look at color. Journal of Philosophy, 93(11), 537-553.

Morrison, J. (2015). Perceptual confidence. Analytic Philosophy, 57(1), 15-48.

Morrison, J. (2019). Third-personal evidence for perceptual confidence. Unpublished manuscript.

Moss, S. (2019). Probabilistic knowledge. Oxford: Oxford University Press.

Munton, J. (2016). Visual confidence and perceptual justification. Philosophical Topics, 44(2), 301-326.

Nanay, B. (2020). Perceiving indeterminately. Thought: A Journal of Philosophy, 9(3), 160-166. https://doi.org/10. $1002 /$ tht 3.454

O'Callaghan, C., Kveraga, K., Shine, J., Adams, R. \& Bar, M. (2017). Predictions penetrate perception: Converging insights from brain, behaviour and disorder. Consciousness and Cognition, 47, 63-74.

Perky, C. (1910). An experimental study of imagination. American Journal of Psychology, 21(3), 422-452.

Pohl, S. (2019). Perceptual representations are not probabilistic. New York University. Unpublished manuscript.

Rahnev, D. (2017) The case against full probability distributions in perceptual decision making. bioRxiv 20, 108944.

Raleigh, T. \& Vindrola, F. (n.d.). Perceptual experience and degrees of belief. The Philosophical Quarterly forthcoming.

Ramsey, F. (1926/2010). Truth and probability. In A. Eagle (Ed.), Philosophy of probability: Contemporary readings (pp. 52-94). London: Routledge. 
Schacter, D. L. \& Dodson, C. S. (2001). Misattribution, false recognition and the sins of memory. Philosophical Transactions of the Royal Society of London. Series B, Biological Sciences, 356(1413), 1385-1393. https://doi. org/10.1098/rstb.2001.0938

Shea, N. (2018). Representation in cognitive science. Oxford: Oxford University Press.

Shea, N. \& Frith, C. (2019). The global workspace needs metacognition. Trends in Cognitive Science, 23(7), 560571. https://doi.org/10.1016/j.tics.2019.04.007.

Shi, L., Griffiths, T., Feldman, N. \& Sanborn, A. (2010). Exemplar models as a mechanism for performing Bayesian inference. Psychonomic Bulletin \& Review, 17(4), 443-464. https://doi.org/10.3758/PBR.17.4.443

Siegel, S. (2017). The rationality of perception. Oxford: Oxford University Press.

Silins, N. (2011). Seeing through the "veil of perception". Mind, 120(478), 329-367.

Smith, A. D. (2008). Translucent experiences. Philosophical Studies, 140(2), 197-212.

Sturgeon, S. (2020). The rational mind. Oxford: Oxford University Press.

Trommershäuser, J., Kording, K. \& Landy, M. (Eds.) (2011). Sensory cue integration. Oxford: Oxford University Press.

van Bergen, R. S., Ma, W., Pratt, M. \& Jehee, J. (2015). Sensory uncertainty decoded from visual cortex predicts behavior. Nature Neuroscience, 18, 1728-1730. https://doi.org/10.1038/nn.4150

Vance, J. (2020). Precision and perceptual clarity. Australasian Journal of Philosophy. https://doi.org/10.1080/ 00048402.2020 .1767663

Walker, E. Y., Cotton, R. J., Ma, W. J. \& Tolias, A. S. (2020). A neural basis of probabilistic computation in visual cortex. Nature Neuroscienc, 23, 122-129.

Watzl, S. (2017). Structuring mind: The nature of attention and how it shapes consciousness. Oxford: Oxford University Press.

Wegner, D. (2002). The illusion of conscious will. Cambridge, MA: MIT Press.

Weisberg, J. (2009). Commutativity or holism? A dilemma for conditionalizers. British Journal for the Philosophy of Science, 60(4), 793-812.

Weiss, Y., Simoncelli, E. \& Adelson, E. (2002). Motion illusions as optimal percepts. Nature Neuroscience, 5, 598-604.

How to cite this article: Siegel S. How can perceptual experiences explain uncertainty? Mind \& Language. 2020;1-25. https://doi.org/10.1111/mila.12348 